\title{
Separation of Different Types of Monosaccharides by Polyacrylamide Column Chromatography
}

\author{
Slavomír Bystrický, Izabela Medovarská, and Eva Machová \\ Institute of Chemistry, GLYCOMED Centre, Slovak Academy of Sciences, 84538 Bratislava, \\ Slovakia \\ Reprint requests to Eva Machová. Phone: +421 25941 0221. E-mail: chememch@savba.sk
}

Z. Naturforsch. 2011, 66b, 295 -298; received December 2, 2010

\begin{abstract}
A high-throughput polyacrylamide gel chromatography was effectively tested to separate different types of monosaccharides. These occur for example in the complex bacterial LPS (lipopolysaccharide) structures as well as in other compounds. Here, a Bio-Gel P-2 column eluted only with water at ambient temperature afforded a convenient separation of the main structural types of neutral, charged, or highly polar hexoses, as well as $N$-acetylated hexose derivatives. These can be easily isolated on the semi-preparative scale by this method. The method was evaluated with a mixture of all basic types of monosaccharides, and repeatedly an efficient resolution was achieved. As an example of a complex structure, the monosaccharide constituents of the delipidated bacterial lipopolysaccharides (dLPS) of V. cholerae $\mathrm{O} 135$ were effectively separated after acid hydrolysis.
\end{abstract}

Key words: Polyacrylamide Chromatography, Monosaccharides, Separation

\section{Introduction}

Structure determination of bacterial polysaccharides (capsular or lipopolysaccharides - LPS) is not an easy task. Measurement of their NMR spectra may not be enough and resolution and complete NMR signal assignment may remain a challenge, and therefore other demanding and time consuming methods (e.g. GCMS) must be employed. This is especially true when the polysaccharide structure consists of many (perhaps more than six) different monosaccharide components in the repeating unit. To solve the problem, we tried to find an effective separation method which would quantitatively and qualitatively separate different types of monosaccharides after acid hydrolysis of the polysaccharide. The method should be simple, reproducible without altering the structure and easy to perform on a preparative scale. The obvious method of choice is liquid chromatography. Weak-ion exchange chromatography can be partially effective for the isolation of positively or negatively charged structures and, in addition, requires a well tuned elution program. Another method is hydrophilic interaction chromatography (HILIC) using silica with bonded polar groups [1-3]. In this method the analytes are eluted in the order of increasing polarity (as opposed to reversed-phase chromatography). The method has been elaborated for analytical HPLC analyses [4]. However, upscaling this method to semi-preparative amounts may lead to problems of reequilibration as well as precise ionic strength consistency. Semi-preparative amounts of purified products, e. g. monosaccharides, are needed for follow-up NMR spectroscopy.

Some authors prepared HPLC monolithic silica capillary columns coated with polyacrylamide and successfully tested them with carbohydrate pyridylamino derivatives [5,6]. Polyacrylamide is an uncharged, strongly hydrophilic material and is commonly used in electrophoresis as well as in gel filtration chromatography.

We explored commercial polyacrylamide beads, namely Bio-Gel P-2, to test the separation of different types of monosaccharides. In this test we used a series of monosaccharides - aldohexose, deoxyhexose, acetylated hexose, hexosamine, and uronic acid to cover the basic natural monosaccharide units. The delipidated LPS of Vibrio cholerae O135 was used for testing of the efficiency of the separation after trifluoroacetic acid hydrolysis of the polysaccharide.

\section{Experimental Section}

Materials

Monosaccharide standards: D-Glucose, L-fucose, Dglucuronic acid, D-galactosamine hydrochloride, and $N$ acetyl-D-glucosamine were purchased from Sigma-Aldrich 


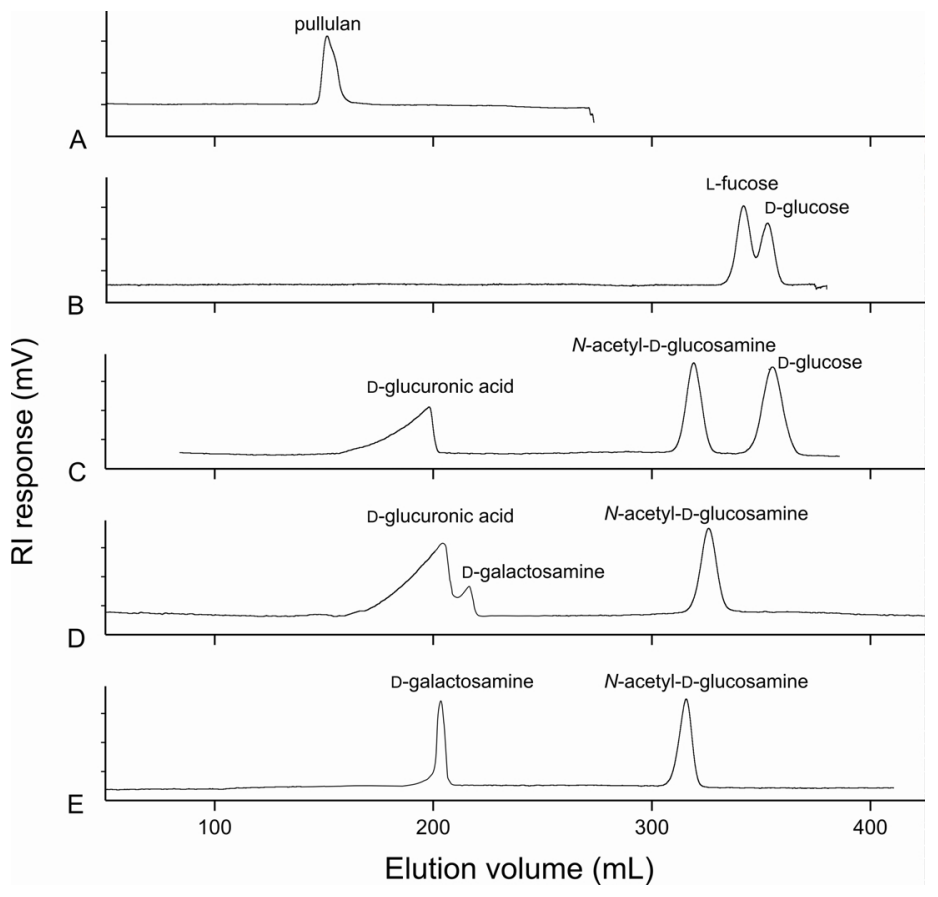

Fig. 1. Separation of different types of monosaccharides by Bio-Gel P-2 polyacrylamide column chromatography with deionized water as the only eluent.
(Germany). Polymeric pullulan standard $(M \mathrm{w}=21100$, Polysaccharide calibration kit PL2090-0100, Lot 13) was purchased from Polymer Laboratories, now a part of Varian Inc. (U. K.). Bio-Gel P-2 fine (hydrated bead size 45$90 \mu \mathrm{m}$ ) was purchased from Bio-Rad (USA). Deionized purified water was obtained from a water purification unit (TQM, So-Safe, Water Quality, USA).

\section{Preparation of a dLPS sample}

An isolate of V. cholerae O135 was obtained from the slovak river Váh near Kolárovo. Bacteria were grown aerobically at $30{ }^{\circ} \mathrm{C}$ in a medium containing $10 \mathrm{~g} \mathrm{~L}^{-1}$ of $\mathrm{NaCl}$ and $10 \mathrm{~g} \mathrm{~L}^{-1}$ of bacterial pepton. Cells were killed with phenol. The LPS of $V$. cholerae $\mathrm{O} 135$ was obtained by extraction of the wet biomass with $90 \%$ phenol in water at $68^{\circ} \mathrm{C}$. Delipidation of polysaccharide was performed by acid hydrolysis. The delipidated polysaccharide was purified by size exclusion chromatography on a Bio-Gel P-30 column as described previously [7]. The obtained dLPS ( $O$-specific chain + core saccharides) was hydrolyzed to its monosaccharide components by the trifluoroacetic acid method. The mixture of monosaccharides after the hydrolysis was applied to a BioGel P-2 column to confirm the separation results obtained with the mixture of commercial monosaccharide standards.

\section{Chromatographic method}

The chromatographic separation was performed on a BioGel P-2 fine chromatography column. The chromatogra- phy medium was a co-polymer of acrylamide and $N, N^{\prime}$ methylene-bis-acrylamide. The chromatographic equipment consisted of the peristaltic pump (Masterflex C/L, Cole Parmer, USA), the column $\left(2 \times 150 \mathrm{~cm}^{2}\right)$, and the refractometer RI-101 (Shodex, Japan). Deionized purified water was used as the sole mobile phase at a flow rate of $\sim 10 \mathrm{mLh}^{-1}$ at ambient temperature. First, individual monosaccharide standards $(10 \mathrm{mg} / 3 \mathrm{~mL})$ were applied to the column and their retention times noted. After that, different combinations of two and three standard mixtures were applied to the column at identical conditions $(10 \mathrm{mg}$ of each component in $3 \mathrm{~mL}$ of water). To test the reproducibility, the separation was repeated three times. The eluted monosaccharides were also identified by NMR spectroscopy.

\section{Results and Discussion}

Unexpectedly, a good separation of mixtures of different types of monosaccharides was repeatedly and reproducibly obtained by using our setup. The resultant chromatograms are shown in Fig. 1. The results indicate that the least polar (e.g. neutral) monosaccharides are retained on the column in size exclusion mode (e.g. in gel filtration mode). They probably enter the bead pores and are eluted according to their molecular size (Fig. 1b). As an example of this separation mode, a good separation of mannooligomers on such polyacrylamide column was documented recently [8]. On 


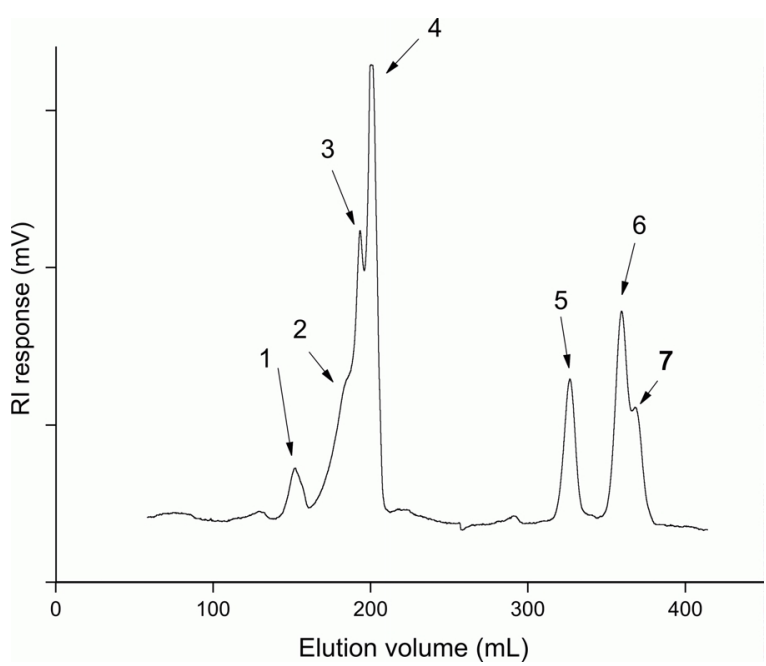

Fig. 2. Elution profile of monosaccharides obtained after TFA hydrolysis of dLPS from V. cholerae O135.

the other side, we found that charged and highly polar monosaccharides are strongly repelled by the column beads (Figs. 1c-e). The reason for this could be the chemical structure of polyacrylamide. The amide groups on the polyacrylamide probably represent an electrostatic barrier for the entrance of the highly polar molecules into the bead pores. Therefore charged uronic monosaccharides as well as polar amino derivatives are eluted in the first fractions from monosaccharide mixtures. That allows an efficient isolation of these derivatives. Indeed, water, as the mobile phase, creates hydration layers on both the stationary phase and the monosaccharides. The active water exchange participates in the dynamic equilibrium during the interactions in the separation system. The effective hydrated size of the molecules plays a role in the elution rate of such small molecules. The increased size of $\mathrm{N}$-acetylated monosaccharides and the repulsion of their amide groups from the polyacrylamide gel cause earlier elution of $N$-acetylated derivatives compared to simple underivatized monosaccharides.

To test the results with a complex polysaccharide structure, we chose the delipidated LPS (dLPS) containing only the saccharide part of LPS without the lipid A. V. cholerae non-O1 structures belong to the most complicated LPSs. As a good example, the well known pathogenic strain $V$. cholerae $\mathrm{O} 139$ contains six different monosaccharide residues in its repeating unit (an uronic acid, $N$-acetylated saccharides and 6-deoxysaccharides).

Our V. cholerae $\mathrm{O} 135$ strain was a new isolate without detailed structural characterization. A preliminary analysis revealed a highly complex structure [7]. The monosaccharide mixture, obtained after acid hydrolysis of the dLPS was applied to the tested polyacrylamide column. The resulting chromatogram is displayed in Fig. 2. There are four major elution peak groups clearly resolved. The first peak was identified as the mixture of partially unhydrolyzed oligosaccharides. The second group contains at least three peaks each belonging to different monosaccharides. We found that peak 2 of this group contains predominantly amino-deoxysaccharides, peak 3 contains glucuronic acid, and peak 4 contains the amino-saccharides glucosamine and mannosamine. The next well-separated group (peak 5) contains $N$-acetylated monosaccharides, $N$-acetyl-D-glucosamine and $N$-acetyl-Dmannosamine. The last separately eluted group of peaks (peaks 6 and 7) contains neutral monosaccharides, mainly glucose, mannose and galactose.

\section{Conclusions}

For the first time a complex an aqueous monosaccharide mixture was separated by a simple, commonly used, inexpensive and robust polyacrylamide chromatography method on a semi-preparative scale. The method allows good separation of different structure types of monosaccharides and could be a very efficient starting point for structure analysis of complex polysaccharides.

\section{Acknowledgements}

This work was supported by the Slovak Research and Development Agency (APVV 0032-06 and APVV-LPP-019409) and VEGA 2/0040/10. The authors are grateful to Dr. M. Matulová for checks of fractions by NMR spectroscopy.
[1] A. J. Alpert, M. Shukla, A. K. Shukla, L. R. Zieske, S. W. Yuen, M. A. J. Ferguson, A. Mehlert, M. Pauly, R. J. Orlando, J. Chromatogr. A 1994, 676, $191-202$.

[2] A. J. Alpert, Anal. Chem. 2008, 80, 62-76.
[3] G. Karlsson, S. Winge, H. Sandberg, J. Chromatogr. A 2005, 1092, 246-249.

[4] G. Karlsson, A. C. Hinz, S. Winge, J. Chromatogr. Sci. 2004, 42(7), 361-365. 
[5] T. Ikegami, H. Fujita, K. Horie, K. Hosoya, N. Tanaka, Anal. Bioanal. Chem. 2006, 386, 578-585.

[6] K. Horie, T. Ikegami, K. Hosoya, N. Saad, O. Fiehn, N. Tanaka, J. Chromatogr. A. 2007, 1164, 198-205.

[7] J. Korcová, E. Machová, P. Farkaš, S. Bystrický, Bi- ologia (Section Cellular and Molecular Biology) 2010 65/5, 1-8. DOI: 10.2478/s11756-010-0092-9.

[8] I. Ližičárová, M. Matulová, P. Capek, E. Machová, Carbohydr. Polym. 2007, 70, 89-100. 